\title{
THE SOLUTION OF AN OPEN PROBLEM GIVEN BY H. HARUKI AND T.M. RASSIAS
}

\author{
BARA KIM \\ Korea Advanced Institute of Science and Technology (KAIST) \\ Department of Mathematics and Center for Applied Mathematics \\ 373-1 Kusong-Dong, Yusong-Gu, Taejon 305-701, Korea \\ e-mail: bara@mathx.kaist.ac.kr
}

(Received July, 1998; Revised December, 1998)

Haruki and Rassias [1] generalized the Poisson kernel in two dimensions and discussed integral formulas for each case. They presented an open problem for an integral formula. In this paper, we give a solution to that problem.

Key words: Poisson Kernel, Integral Formula.

AMS subject classifications: $31 \mathrm{~A} 05,31 \mathrm{~A} 10$.

\section{Introduction}

Haruki and Rassias [1] introduced two types of generalizations of the Poisson kernel. One of them is defined by

$$
Q(\theta ; a, b) \triangleq \frac{1-a b}{\left(1-a e^{i \theta}\right)\left(1-b e^{-i \theta}\right)}
$$

where $a, b$ are complex parameters satisfying $|a|<1$ and $|b|<1$.

They proved the integral formulas:

$$
\begin{gathered}
\frac{1}{2 \pi} \int_{0}^{2 \pi} Q(\theta ; a, b) d \theta=1, \\
\frac{1}{2 \pi} \int_{0}^{2 \pi} Q(\theta ; a, b)^{2} d \theta=\frac{1+a b}{1-a b} .
\end{gathered}
$$
"Let

They set the open problem as follows: 


$$
\begin{gathered}
I_{n} \triangleq \frac{1}{2 \pi} \int_{0}^{2 \pi} Q(\theta ; a, b)^{n+1} d \theta \\
=\frac{1}{2 \pi} \int_{0}^{2 \pi}\left(\frac{1-a b}{\left(1-a e^{i \theta}\right)\left(1-b e^{-i \theta}\right)}\right)^{n+1} d \theta, \quad(n=0,1, \ldots),
\end{gathered}
$$

where $a, b$ are complex parameters satisfying $|a|<1$ and $|b|<1$. Compute $I_{n}$ for $n=2,3,4, \ldots . . "$

In the next section, we will give the solution to the problem.

\section{Solution of the Problem}

Theorem 1: $I_{n}$, defined by (3), satisfies

$$
I_{n}=\sum_{j=0}^{n} \frac{(2 n-j) !}{j !((n-j) !)^{2}}\left(\frac{a b}{1-a b}\right)^{n-j}
$$

for $n=0,1,2, \ldots$, and complex values $a, b$ are such that $|a|<1$ and $|b|<1$.

Proof: By the change of variables, with $z=e^{i \theta},(3)$ becomes

Let

$$
\begin{aligned}
I_{n} & =\frac{1}{2 \pi i} \oint_{|z|=1}\left(\frac{1-a b}{(1-a z)\left(1-b z^{-1}\right)}\right)^{n+1} z^{-1} d z \\
& =\frac{1}{2 \pi i} \oint_{|z|=1}\left(\frac{1-a b}{1-a z}\right)^{n+1} z^{n}(z-b)^{-n-1} d z .
\end{aligned}
$$

$$
f(z) \triangleq\left(\frac{1-a b}{1-a z}\right)^{n+1} z^{n}(z-b)^{-n-1} .
$$

Then $f(z)$ is analytic on $\{z \in \mathbb{C}:|z| \leq 1, z \neq b\}$ and has a pole at $z=b$. Therefore, by the residue theorem, $I_{n}$ is the residue of $f(z)$ at $z=b$.

The Laurent series expansion of $f(z)$ at $z=b$ gives:

$$
\begin{gathered}
f(z)=\left(\frac{1}{1-\frac{a}{1-a b}(z-b)}\right)^{n+1}(b+(z-b))^{n}(z-b)^{-n-1} \\
=\sum_{k=0}^{\infty}\left(\begin{array}{c}
n+k \\
k
\end{array}\right)\left(\frac{a}{1-a b}\right)^{k}(z-b)^{k} \sum_{j=0}^{n}\left(\begin{array}{c}
n \\
j
\end{array}\right) b^{n-j}(z-b)^{j}(z-b)^{-n-1} \\
=\sum_{k=0}^{\infty} \sum_{j=0}^{n}\left(\begin{array}{c}
n+k \\
k
\end{array}\right)\left(\begin{array}{c}
n \\
j
\end{array}\right)\left(\frac{a}{1-a b}\right)^{k} b^{n-j}(z-b)^{k+j-n-1} .
\end{gathered}
$$

Therefore, the residue of $f(z)$ at $b$, which is $I_{n}$, is given by 


$$
\begin{aligned}
I_{n} & =\sum_{j=0}^{n}\left(\begin{array}{c}
2 n-j \\
n-j
\end{array}\right)\left(\begin{array}{c}
n \\
j
\end{array}\right)\left(\frac{a b}{1-a b}\right)^{n-j} \\
& =\sum_{j=0}^{n} \frac{(2 n-j) !}{j !((n-j) !)^{2}}\left(\frac{a b}{1-a b}\right)^{n-j} .
\end{aligned}
$$

Note that we obtain (1) and (2) by substituting $n=0$ and $n=1$, respectively.

\section{References}

[1] Haruki, H. and Rassias, T.M., New generalizations of the Poisson kernel, J. Appl. Math. Stoch. Anal. 10:2 (1997), 191-196. 


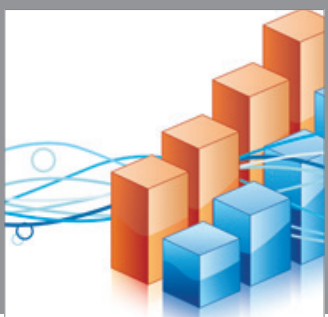

Advances in

Operations Research

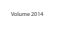

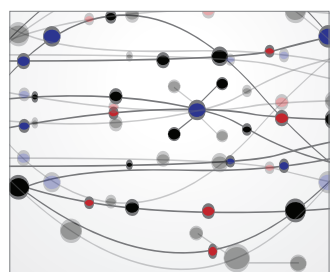

\section{The Scientific} World Journal
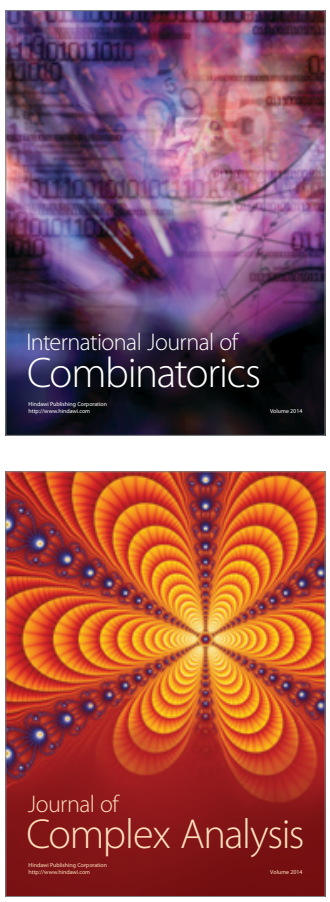

International Journal of

Mathematics and

Mathematical

Sciences
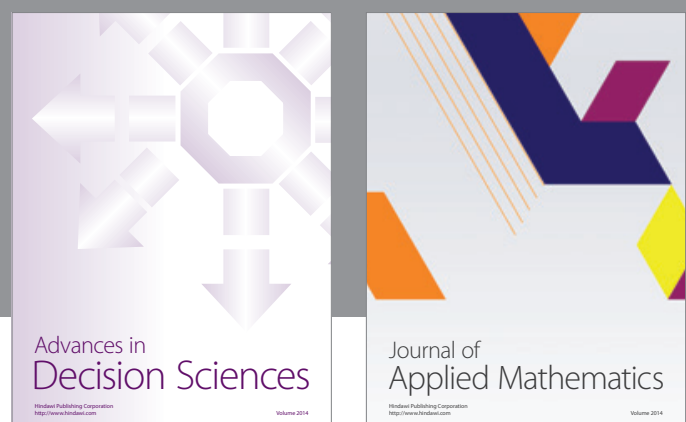

Journal of

Applied Mathematics
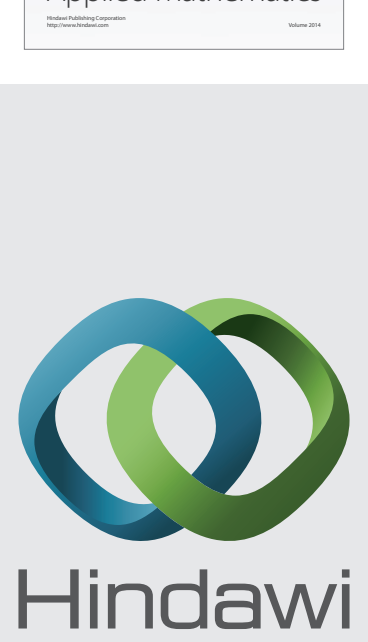

Submit your manuscripts at http://www.hindawi.com
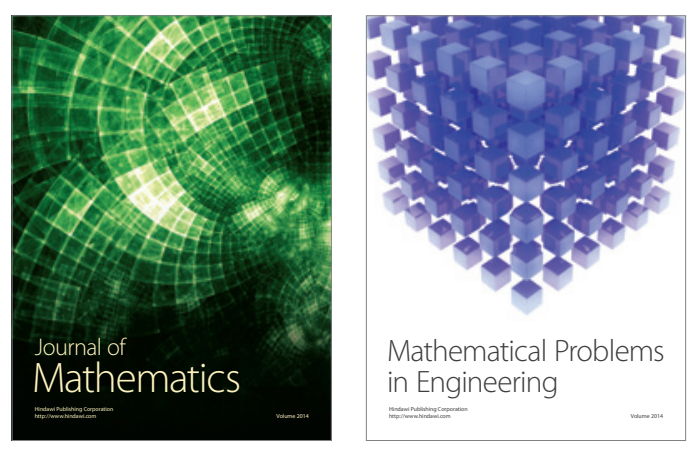

Mathematical Problems in Engineering
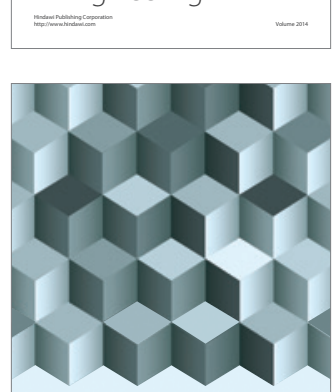

Journal of

Function Spaces
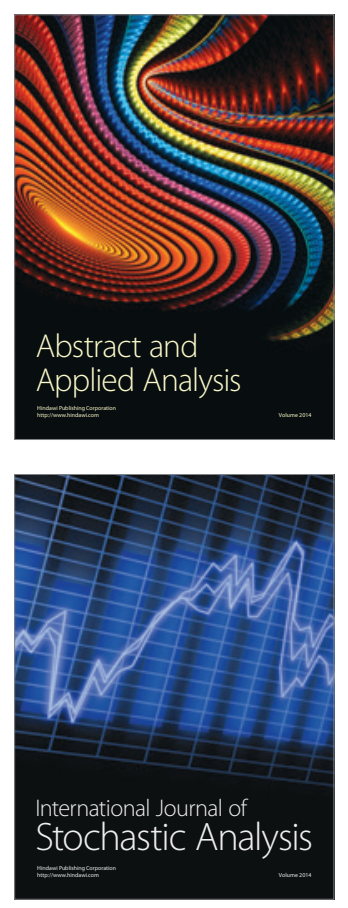

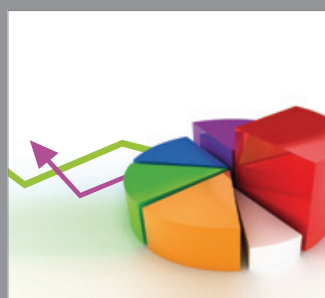

ournal of

Probability and Statistics

Promensencen
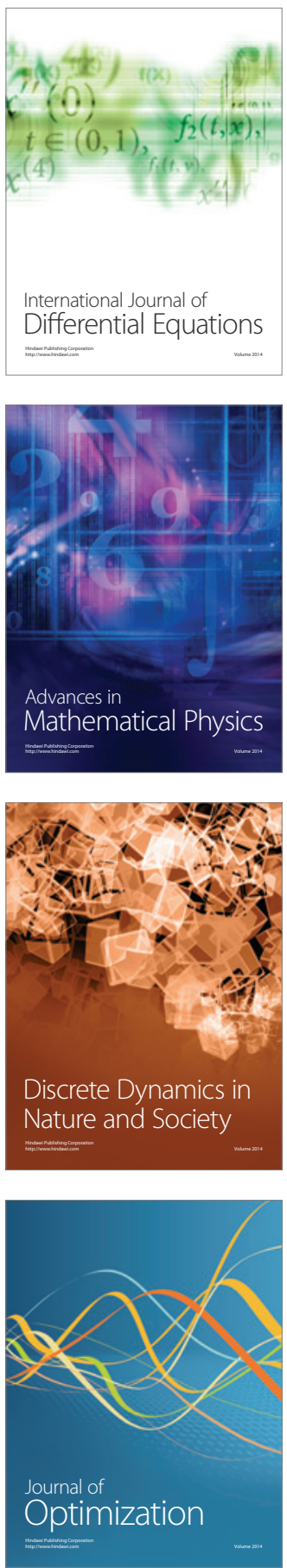